\title{
CMMSE: Study of a new symmetric anomaly in the elliptic, hyperbolic and parabolic Keplerian motion
}

\author{
jose antonio lópez ortí ${ }^{1}$, Vicemte Agost Gómez ${ }^{1}$, and Miguel Barreda rochera ${ }^{1}$ \\ ${ }^{1}$ Universitat Jaume I
}

November 30, 2021

\begin{abstract}
In the present work, we define a new anomaly, $\$ \backslash P s i \$$, termed semifocal anomaly. It is determined by the mean between the true anomaly, $\$ \mathrm{f} \$$, and the antifocal anomaly, $\$ \mathrm{f}^{\wedge}\{\backslash$ prime $\}$; Fukushima defined $\$ \mathrm{f}^{\wedge}\{\backslash$ prime $\} \$$ as the angle between the periapsis and the secondary around the empty focus. In this first part of the paper, we take an approach to the study of the semifocal anomaly in the hyperbolic motion and in the limit case correspoding to the parabolic movement. From here we find a relation beetween the semifocal anomaly and the true anomaly that holds independently of the movement type. We focus on the study of the two-body problem when this new anomaly is used as the temporal variable. $\backslash \backslash$ In the second part, we show the use of this anomaly — combined with numerical integration methods - to improve integration errors in one revolution. Finally, we analyze the errors committed in the integration process — depending on several values of the eccentricity - for the elliptic, parabolic and hyperbolic cases in the apsidal region.
\end{abstract}

\section{Hosted file}

lopez_CMMSE_2021_MMAS_final.pdf available at https://authorea.com/users/448674/articles/ 547433-cmmse-study-of-a-new-symmetric-anomaly-in-the-elliptic-hyperbolic-and-parabolickeplerian-motion 
figures/E-H/E-H-eps-converted-to.pdf 
figures/semifocal/semifocal-eps-converted-to.pdf 